\title{
Approximation on the rotation group $\mathrm{SO}(3)$
}

\author{
Zhuyuan Yang ${ }^{\mathrm{a}, *}$, Xin Wang ${ }^{\mathrm{a}}$, Xinzhi Liu \\ ${ }^{a}$ School of Mathematics and Computer Science, Yunnan Minzu University, Kunming 650500, P. R. China. \\ ${ }^{b}$ Department of Mathematics, University of Waterloo, Waterloo, Canada N2L $3 G 1$.
}

Communicated by R. Saadati

\begin{abstract}
In this paper we study the approximation on rotation group $\mathrm{SO}(3)$, we consider the partial sum, Féjer and Jackson-type operators and obtain the approximation theorems in $L_{p}(1 \leqslant p \leqslant+\infty)$ respectively. (C)2017 All rights reserved.
\end{abstract}

Keywords: Rotation group, operator, approximation.

2010 MSC: 41A25, 41A35, 41A60.

\section{Introduction}

Many results of approximation are based on Euclid spaces or their compact subsets. Periodic approximation is based on compact group $\{\exp (i x)\}$, whereas matrix group $U(n)$ is the generalization of $\{\exp (i x)\}$. We know homomorphism between $\mathrm{SU}(2)$ and rotation group $\mathrm{SO}(3)$, which has many applications in Physics and Chemistry. Some approximation problems on compact groups have been studied since in 1920s Peter and Weyl [7] proved the approximation theorem on compact group, that is, the irreducible character generates a dense subspace of the space of continuous classes function. For instance, Gong [2] studied the basic problems of Fourier analysis on unitary and rotation groups, including the degree of convergence of Abel sum based on Poisson kernel. Zheng et al. (see [11, 12]) studied the polynomial approximation on compact Lie groups. Cartwright et al. [1] studied Jackson's theorem for compact connected Lie groups, and so on. No matter from the results or research methods, the approximation on compact Lie groups is different from on the classical cases, Eulid spaces. For example, Riemann-Lebesgue lemma is not necessarily true in $L_{p}(G)(1 \leqslant p \leqslant 4 / 3)$, with $G$ being a compact Lie group (see [8]). In this paper, we study the approximation of the partial sums, Féjer and Jackson-type operators on $\mathrm{SO}(3)$ in $\mathrm{L}_{\mathrm{p}}(1 \leqslant \mathrm{p} \leqslant+\infty)$.

Let $\mathrm{G}=\mathrm{SO}(3)=\left\{\mathbf{x} \in \mathrm{GL}(3, \mathbb{R}), \mathbf{x}^{\top} \mathbf{x}=\mathbf{E}\right.$, $\left.\operatorname{det} \mathbf{x}=1\right\}$ be the rotation group, where $\mathrm{GL}(\mathrm{n}, \mathbb{R})$ is the group of invertible real $(n \times n)$-matrices. For $1 \leqslant p<+\infty, L_{p}(G)=\left\{f:\|f\|_{p}=\left[\int_{G}|f(x)|^{p} d \mu(x)\right]^{1 / p}<+\infty\right\}$, and

\footnotetext{
*Corresponding author

Email addresses: yangzhuyuan@sina.com (Zhuyuan Yang), wxkmyn@163.com (Xin Wang), xinzhi.liu@uwarterloo.ca (Xinzhi Liu)
}

doi:10.22436/jnsa.010.04.22 
$\mu$ is the normalized Harr measure on $G$, conveniently, we write $d \mu(\mathbf{x})$ as $d \mathbf{x}$ in this paper. When using Euler angles, the Harr integral of a function $\mathrm{f}$ on $\mathrm{SO}(3)$ reads as

$$
\int_{S O(3)} f(x) d \mu(\mathbf{x})=\frac{1}{8 \pi^{2}} \int_{0}^{2 \pi} d \alpha \int_{0}^{\pi} \sin \beta d \beta \int_{0}^{2 \pi} d \gamma f(\alpha, \beta, \gamma)
$$

An element $\mathbf{x} \in \mathrm{SO}(3)$ is identified with a point in the projective spaces $S_{\pi}$ of the closed ball in $\mathbb{R}^{3}$ of radius $\pi$ by $\mathbf{x} \rightarrow \omega \mathbf{r}$, satisfying $\mathbf{x r}=\mathbf{r}$, and $\|\mathbf{r}\|=1$, where $\mathbf{r}$ is the rotation axis and $\omega \in[0, \pi]$ is the rotation angle of $\mathbf{x} \cdot(\phi, \theta, \varphi)$ denotes the direction angles of rotation axis $\mathbf{r}$ and $\omega$ the rotation angle, then there hold following formulas (see [9]) between $(\mathbf{r}, \omega)$ and Euler angles $(\alpha, \beta, \gamma)$,

$$
\cos \frac{\omega}{2}=\cos \frac{\beta}{2} \cos \frac{\alpha+\gamma}{2}, \phi=-\frac{\sin \frac{\beta}{2} \sin \frac{\alpha-\gamma}{2}}{\sin \frac{\omega}{2}}, \theta=\frac{\sin \frac{\beta}{2} \cos \frac{\alpha-\gamma}{2}}{\sin \frac{\omega}{2}}, \phi=\frac{\cos \frac{\beta}{2} \sin \frac{\alpha+\gamma}{2}}{\sin \frac{\omega}{2}} .
$$

We know a function $\mathrm{f}$ on $\mathrm{SO}(3)$ only depends on the rotation angle of the argument coincide exactly with the class function-functions that are conjugacy classes, i.e., $f(\mathbf{x})=f\left(\mathbf{y x y}^{-1}\right)$ for all $\mathbf{x}, \mathbf{y} \in \mathrm{SO}(3)$. In other words, for any function $f$ on $S O(3)$ there is a uniquely determined $\tilde{f}:[0, \pi] \rightarrow \mathbb{C}$ such that $f(\mathbf{x})=\tilde{f}(\omega(\mathbf{x}))$. The Haar integral for such a class function reads as

$$
\int_{S O(3)} f(\mathbf{x}) \mathrm{d} \mu(\mathbf{x})=\int_{\operatorname{SO}(3)} \tilde{f}(\omega(\mathbf{x})) \mathrm{d} \mu(\mathbf{x})=\frac{2}{\pi} \int_{0}^{\pi} \tilde{f}(\omega) \sin ^{2} \frac{\omega}{2} \mathrm{~d} \omega .
$$

We make the convention that if $\mathrm{f}$ is a class function on $\mathrm{SO}(3)$ and if no confusion occurs, we subsequently drop the tilde. Denote by

$$
S_{N} f(\alpha, \beta, \gamma)=\sum_{l=0}^{N} \sum_{m, n=-l}^{l} \sqrt{2 l+1} C_{m, n}^{l} D_{m, n}^{l}(\alpha, \beta, \gamma),
$$

the partial sum operators, where $(\alpha, \beta, \gamma) \in[0,2 \pi] \times[0, \pi] \times[0,2 \pi]$ are Euler angles and

$$
C_{m, n}^{l}=\frac{\sqrt{2 l+1}}{8 \pi^{2}} \int_{0}^{2 \pi} d \alpha \int_{0}^{\pi} \sin \beta d \beta \int_{0}^{2 \pi} d \gamma f(\alpha, \beta, \gamma) \overline{D_{m, n}^{l}(\alpha, \beta, \gamma)},
$$

and for $\mathbf{x} \in \mathrm{SO}(3)$,

$$
D_{m, n}^{l}(\mathbf{x})=D_{m, n}^{l}(\alpha, \beta, \gamma)=e^{-i m \alpha} P_{m, n}^{l}(\cos \beta) e^{-i n \gamma},-l \leqslant m, n \leqslant l,
$$

the function $\mathrm{P}_{\mathrm{m}, \mathrm{n}}^{\mathrm{l}}$ is given by

$$
P_{m, n}^{l}(u)=C(1-u)^{\frac{n-m}{2}}(1+u)^{-\left(\frac{n+m}{2}\right)} \frac{d^{l-m}}{d u^{l-m}}\left[(1-u)^{l-n}(1+u)^{l+n}\right],
$$

with $C=\frac{(-1)^{l-n_{i^{n-m}}}}{2^{l}} \sqrt{\frac{(l+m) !}{(l-n) !(l+n) !(l-m) !}}$.

\section{Theorems and their proofs}

We start with the partial sum operator,

$$
\begin{aligned}
S_{N}(f, x) & =S_{N} f(\alpha, \beta, \gamma) \\
& =\sum_{l=0}^{N} \sum_{m, n=-l}^{l} \sqrt{2 l+1} C_{m, n}^{l} D_{m, n}^{l}(\alpha, \beta, \gamma)
\end{aligned}
$$




$$
\begin{aligned}
& =\sum_{l=0}^{N} \sum_{m, n=-l}^{l} \frac{2 l+1}{8 \pi^{2}} \int_{0}^{2 \pi} d \zeta \int_{0}^{\pi} \sin \eta d \eta \int_{0}^{2 \pi} d \xi f(\zeta, \eta, \xi) \overline{D_{m, n}^{l}(\zeta, \eta, \xi)} D_{m, n}^{l}(\alpha, \beta, \gamma) \\
& =\sum_{l=0}^{N} \frac{2 l+1}{8 \pi^{2}} \int_{0}^{2 \pi} d \zeta \int_{0}^{\pi} \sin \eta d \eta \int_{0}^{2 \pi} d \xi f(\zeta, \eta, \xi) \sum_{m, n=-l}^{l} \overline{D_{m, n}^{l}(\zeta, \eta, \xi)} D_{m, n}^{l}(\alpha, \beta, \gamma) \\
& =\sum_{l=0}^{N}(2 l+1) \int_{S O(3)} f(g) \operatorname{Tr}\left(D^{l}\left(g^{-1} \mathbf{x}\right)\right) d g \\
& =\sum_{l=0}^{N}(2 l+1) f * \chi_{l}(\mathbf{x})=f * D_{N}(\mathbf{x}),
\end{aligned}
$$

where $D^{l}(\mathbf{x})=\operatorname{matrix}\left(D_{m, n}^{l}\right)$ is the $(2 l+1)$-dimensional irreducible representation of $G, \chi_{l}(\mathbf{x})=\operatorname{Tr}\left[D^{l}(\mathbf{x})\right]$ is the character of irreducible representation, $D_{N}(\mathbf{x})=\sum_{l=0}^{N}(2 l+1) \chi_{l}(\mathbf{x})$ is Dirichlet kernel, and the convolution of two functions $f$ and $g$ is defined by

$$
(f * g)(\mathbf{x})=\int_{G} f(g) g\left(g^{-1} \mathbf{x}\right) d \mathbf{g} .
$$

Next orthogonal properties of $\mathrm{D}^{l}$ and $\chi_{l}$ are well-known (see [3]),

$$
\begin{aligned}
\frac{2 j_{1}+1}{8 \pi^{2}} \int_{0}^{2 \pi} \mathrm{d} \alpha \int_{0}^{\pi} \sin \beta d \beta \int_{0}^{2 \pi} & d \gamma \overline{D_{m_{1}, n_{1}}^{j_{1}}(\alpha, \beta, \gamma)} D_{m_{2}, n_{2}}^{j_{2}}(\alpha, \beta, \gamma)=\delta_{j_{1}, j_{2}} \delta_{m_{1}, m_{2}} \delta_{n_{1}, n_{2},} \\
\frac{1}{\pi} \int_{0}^{\pi} \overline{\chi_{j_{1}}(\omega)} \chi_{j_{2}}(\omega)(1-\cos \omega) d \omega & =\delta_{j_{1}, j_{2}} .
\end{aligned}
$$

Furthermore, we discuss the properties of Dirichlet kernel $D_{N}$.

Noting that $\chi_{\mathrm{l}}(\mathbf{x})=\operatorname{Tr}\left[\mathrm{D}^{\mathrm{l}}(\mathbf{x})\right]=\frac{\sin (2 \mathrm{l}+1) \frac{\omega}{2}}{\sin \frac{\omega}{2}}$ with $\omega$ as (1.1) being the rotation angle of $\mathbf{x}$ and using Euler formula $e^{i \theta}=\cos \theta+i \sin \theta$, by calculation, we can get the following.

\section{Lemma 2.1.}

$$
\begin{aligned}
D_{N}(x) & =\frac{\sin (N+1) \omega \cdot \cos \frac{\omega}{2}}{2 \sin ^{3} \frac{\omega}{2}}-\frac{(N+1) \cdot \cos (N+1) \omega}{\sin ^{2} \frac{\omega}{2}}, \\
\left\|D_{N}\right\|_{L_{1}(S O(3))} & =O(N)+O(\ln N), \quad\left\|S_{N}\right\|=O(N) .
\end{aligned}
$$

For $f \in L_{p}(G), 1 \leqslant p \leqslant+\infty$, we define the best approximation degree by

$$
E_{N}(f)_{p}=\sup _{T_{N} \in \Pi_{N}}\left\|f-T_{N}\right\|_{p}
$$

where $\Pi_{N}$ is the set of trigonometric polynomial of degree at most $N$, which needs not to be an integer, and when $p=+\infty, L_{\infty}(G)$ is replaced by continuous functions space $C(G)$ and the $p$-norm is replaced by sup-norm, we will not repeat this later.

Thanks to the accuracy of $S_{N}$ for the trigonometric polynomial, that is $S_{N}\left(T_{n}\right)=T_{n}(n \leqslant N), T_{n} \in \Pi_{N}$, subsequently we have the following.

Theorem 2.2. If $f \in \mathrm{L}_{p}(\mathrm{SO}(3)), 1 \leqslant p \leqslant \infty$, then

$$
\left\|f-S_{N}(f)\right\|_{p} \leqslant C N \cdot E_{N}(f)_{p}
$$

with $\mathrm{C}>0$ being an absolute constant. 
Further, we consider the Féjer kernel

$$
\mathrm{F}_{\mathrm{N}}(\mathbf{x})=\frac{\mathrm{D}_{0}(\mathbf{x})+\mathrm{D}_{1}(\mathbf{x})+\cdots+\mathrm{D}_{\mathrm{N}}(\mathbf{x})}{\mathrm{N}+1} .
$$

Using Lemma 2.1, we know

$$
F_{N}(x)=\frac{1}{N+1}\left[\frac{\cos \frac{\omega}{2}}{2 \sin ^{3} \frac{\omega}{2}} \sum_{k=0}^{N} \sin (k+1) \omega-\frac{1}{\sin ^{2} \frac{\omega}{2}} \sum_{k=0}^{N}(k+1) \cos (k+1) \omega\right] .
$$

Note that

$$
\sum_{k=0}^{N} \sin (k+1) \omega=\operatorname{Im}\left[\sum_{k=0}^{N}\left(e^{i \omega}\right)^{k+1}\right]=\left(\sin \frac{N+2}{2} \omega\right) \cdot \frac{\sin \left(\frac{N+1}{2} \omega\right)}{\sin \frac{\omega}{2}}
$$

and

$$
\begin{aligned}
& \sum_{k=0}^{N}(k+1) \cos (k+1) \omega \\
& =\operatorname{Im}\left[\sum_{k=0}^{N}\left(e^{i \omega}\right)^{k+1}\right]^{\prime} \\
& =\frac{N+2}{2}\left(\cos \frac{N+2}{2} \omega\right) \cdot \frac{\sin \frac{N+1}{2} \omega}{\sin \frac{\omega}{2}}+\frac{N+1}{2}\left(\cos \frac{N+1}{2} \omega\right) \cdot \frac{\sin \frac{N+2}{2} \omega}{\sin \frac{\omega}{2}}-\frac{1}{2} \cos \frac{\omega}{2} \cdot \frac{\sin \frac{N+1}{2} \omega \cdot \sin \frac{N+2}{2} \omega}{\sin ^{2} \frac{\omega}{2}},
\end{aligned}
$$

we have

$$
\begin{aligned}
\mathrm{F}_{\mathrm{N}}(\mathbf{x}) & =\frac{1}{2(\mathrm{~N}+1)} \frac{\cos \frac{\omega}{2}}{\sin ^{2} \frac{\omega}{2}} \cdot \frac{\sin \frac{N+1}{2} \omega \cdot \sin \frac{N+2}{2} \omega}{\sin ^{2} \frac{\omega}{2}}-\frac{1}{\sin ^{2} \frac{\omega}{2}}\left[\frac{N+2}{2(N+1)}\left(\cos \frac{N+2}{2} \omega\right) \cdot \frac{\sin \frac{N+1}{2} \omega}{\sin \frac{\omega}{2}}\right. \\
& \left.+\frac{1}{2}\left(\cos \frac{N+1}{2} \omega\right) \cdot \frac{\sin \frac{N+2}{2} \omega}{\sin \frac{\omega}{2}}-\frac{1}{2(N+1)} \cos \frac{\omega}{2} \cdot \frac{\sin \frac{N+1}{2} \omega \cdot \sin \frac{N+2}{2} \omega}{\sin ^{2} \frac{\omega}{2}}\right] \\
& :=I_{1}(\omega)+I_{2}(\omega)+I_{3}(t)+I_{4}(\omega) .
\end{aligned}
$$

\section{Lemma 2.3.}

$$
\left\|\mathrm{F}_{\mathrm{N}}\right\|_{\mathrm{L}_{1}(\mathrm{SO}(3))}=\mathrm{O}(\ln \mathrm{N})
$$

From $D_{N}(\mathbf{x})=\sum_{l=0}^{N}(2 l+1) \chi_{l}(\mathbf{x})$, and the orthogonal properties of $D^{l}$ and $\chi_{l}$, we have

$$
\int_{\mathrm{SO}(3)} \mathrm{F}_{\mathrm{N}}(\mathbf{x}) \mathrm{d} \mathbf{x}=1
$$

We consider the next Féjer convolution operator

$$
\sigma_{N}(f, x)=\int_{S O(3)} F_{N}(\mathbf{y}) f\left(y^{-1} \mathbf{x}\right) d \mathbf{y}
$$

Clearly,

$$
\left|f(\mathbf{x})-\sigma_{N}(f, \mathbf{x})\right| \leqslant \int_{S O(3)}\left|f(\mathbf{x})-f\left(\mathbf{y}^{-1} \mathbf{x}\right) \| F_{N}(\mathbf{y})\right| d \mathbf{y} \leqslant \int_{S O(3)} \omega(f,|\mathbf{y}|)\left|F_{N}(\mathbf{y})\right| d \mathbf{y}
$$

where $|y|=\operatorname{dist}(\mathbf{y}, \mathbf{e})$ and $\mathbf{e}$ is the identity element of $\mathrm{SO}(3)$. For $\mathbf{x}, \mathbf{y} \in \mathrm{SO}(3), \operatorname{dist}(\mathbf{x}, \mathbf{y})=\omega\left(\mathbf{y}^{-1} \mathbf{x}\right)=$ $\omega\left(\mathbf{x}^{-1} \mathbf{y}\right)$ is defined as the rotational angle of the rotation $\mathbf{y}^{-1} \mathbf{x}$ (or its inverse $\mathbf{x}^{-1} \mathbf{y}$ ). 
Remark 2.4. The rotational angle of a rotation $\mathbf{x} \in \mathrm{SO}(3)$ is also defined as $|\mathbf{x}|=\arccos \frac{1}{2}[\operatorname{Tr}(\mathbf{x})-1]$, in terms of Euler angles $|\mathbf{x}|=|\mathbf{x}(\alpha, \beta, \gamma)|=2 \arccos \left(\cos \frac{\beta}{2} \cos \frac{\alpha+\gamma}{2}\right)=\omega(\mathbf{x})=\omega$ (see [4]).

Remark 2.5. $\omega(f, t)_{p}=\sup \left\{\left\|f(x)-f\left(y^{-1} \mathbf{x}\right)\right\|_{p}: d(x, y) \leqslant t\right\}(1 \leqslant p \leqslant \infty)$, is the first-order moduli of smoothness. Higher order moduli $\omega_{r}(f, t)$ (see Definition 2.8) will also be employed later. We need only the inequality $\omega_{r}(f, \lambda t)_{p} \leqslant(1+\lambda)^{r} \omega_{r}(f, t)_{p}(r \in \mathbb{N})$, some other properties can be found in $[1,5]$.

From Remarks 2.4 and 2.5 and (1.2) we have

$$
\begin{aligned}
\left|f(x)-\sigma_{N}(f, x)\right| & \leqslant \omega\left(f, \frac{1}{N}\right) \cdot \int_{S O(3)}(1+N|y|)\left|F_{N}(\mathbf{y})\right| d \mathbf{y} \\
& \leqslant \omega\left(f, \frac{1}{N}\right) \cdot \frac{2}{\pi} \int_{0}^{\pi}(1+N \omega)\left|F_{N}(\omega)\right| \sin ^{2} \frac{\omega}{2} d t \\
& =\omega\left(f, \frac{1}{N}\right) \cdot \frac{2}{\pi} \int_{0}^{\pi}(1+N \omega)\left[\left|I_{1}(\omega)\right|+\left|I_{2}(\omega)\right|+\left|I_{3}(\omega)\right|+\left|I_{4}(\omega)\right|\right] \sin ^{2} \frac{\omega}{2} d t .
\end{aligned}
$$

By (2.1) and subdividing the integration interval $[0, \pi]=\left[0, \frac{\pi}{\mathrm{N}+2}\right] \cup\left[\frac{\pi}{\mathrm{N}+2}, \pi\right]$, we get

$$
\int_{0}^{\pi}(1+N \omega)\left|I_{j}(\omega)\right| \sin ^{2} \frac{\omega}{2} d \omega=O(\ln (N)), j=1,2,3,4 .
$$

Thus, by using Lemma 2.3 and (2.2), we have the following result.

Theorem 2.6. If $f \in \mathrm{L}_{p}(\mathrm{SO}(3)), 1 \leqslant p \leqslant+\infty$, then,

$$
\left\|f-\sigma_{N}(f)\right\|_{p}=O\left(\ln (N) \cdot \omega\left(f, \frac{1}{N}\right)\right)_{p}, \quad 1 \leqslant p \leqslant \infty .
$$

Generally, we conclude the result as follows.

Theorem 2.7. If $\mathrm{f} \in \mathrm{L}_{\mathrm{p}}(\mathrm{SO}(3)), 1 \leqslant \mathrm{p} \leqslant+\infty$, and $\mathrm{V}_{\mathrm{N}} \in \Pi_{\mathrm{N}}$ satisfies

(i): $V_{\mathrm{N}} * \mathrm{~T}_{\mathrm{N}}=\mathrm{T}_{\mathrm{N}}, \forall \mathrm{T}_{\mathrm{N}} \in \Pi_{\mathrm{N}}$, (ii): $\left\|\mathrm{V}_{\mathrm{N}}\right\|_{\mathrm{L}_{1}(\mathrm{G})} \leqslant \mathrm{K}$, with $\mathrm{K}$ being an absolute constant, then for $\mathrm{L}_{\mathrm{N}}(\mathrm{f})=\mathrm{V}_{\mathrm{N}} * \mathrm{f}$,

$$
\left\|f-L_{N}(f)\right\|_{p} \leqslant(1+K) E_{N}(f)_{p} .
$$

Proof. The proof is trivial. The kernel $\mathrm{V}_{\mathrm{N}}$, which satisfies the conditions of Theorem 2.7 , can be found in [6].

Next we give the Jackson-type approximation theorem.

We first give the definition of the $r$-th moduli of smoothness of function $f$ on the rotation group.

As classical case, for $\mathbf{x}, \mathbf{h} \in \mathrm{G}=\mathrm{SO}(3)$, let

$$
\left(\Delta_{\mathbf{h}}^{\mathrm{r}} \mathbf{f}\right)(\mathbf{x})=\sum_{j=0}^{r}(-1)^{r-j}\left(\begin{array}{l}
r \\
j
\end{array}\right) f\left(h^{-j} \mathbf{x}\right)
$$

be the $r$-th difference of $f$ at the point $\mathbf{x}$.

Definition 2.8. Let $f \in L_{p}(G)(1 \leqslant p \leqslant \infty)$, for any integer $r \geqslant 1$ and $t>0$, write

$$
\omega_{r}(f, t)_{p}=\sup \left\{\left\|\Delta_{\mathbf{h}}^{r} f\right\|_{p}: \mathbf{h} \in G, d(\mathbf{e}, \mathbf{h})=|\mathbf{h}| \leqslant t\right\} .
$$

We can also write

$$
\omega_{r}(f, t)_{p}=\sup \left\{\left\|\Delta_{\text {exp }}^{r} f\right\|_{p}: \mathbf{H} \in \mathbf{g},\|\mathbf{H}\| \leqslant t\right\},
$$

where $\mathfrak{g}$ is the Lie algebras of $\mathrm{G}$, and $\|\cdot\|$ is the norm induced by Killing inner product $\leftrightarrow$ on $\mathbf{g}$ (see [10]). 
Let $\mathrm{T}$ be a maximal torus of $\mathrm{SO}(3)$ given by (see [7])

$$
T=\left\{\left(\begin{array}{ccc}
\cos \omega & \sin \omega & 0 \\
-\sin \omega & \cos \omega & 0 \\
0 & 0 & 1
\end{array}\right) \mid \omega \in \mathbb{R} / 2 \pi \mathbb{Z}\right\},
$$

and $\mathfrak{t}$ denotes its respective Lie algebras also called Cartan subalgebras, in fact given by

$$
\mathfrak{t}=\left\{\left(\begin{array}{ccc}
0 & \omega & 0 \\
-\omega & 0 & 0 \\
0 & 0 & 0
\end{array}\right) \mid \omega \in \mathbb{R} / 2 \pi \mathbb{Z}\right\} .
$$

For $\mathbf{u} \in \mathrm{SO}(3)$, let $\phi_{\mathrm{N}, \mathrm{s}}(\mathbf{u})=\lambda_{\mathrm{N}, \mathrm{s}}^{-1}\left[\frac{\sin \left(\mathrm{N}+\frac{1}{2}\right) \omega}{\sin \frac{1}{2} \omega}\right]^{2 \mathrm{~s}}=\phi_{\mathrm{N}, \mathrm{s}}(\omega)$, satisfying $\int_{\mathrm{SO}(3)} \phi_{\mathrm{N}, \mathrm{s}}(\mathbf{u}) \mathrm{d} \mathbf{u}=1$, where $\omega=\omega(\mathbf{u})$ is the rotation angle of $\mathbf{u}$, and $s \geqslant 2+r / 2$.

As the classical case, we have $\lambda_{\mathrm{N}, \mathrm{s}}=\mathrm{O}\left(\mathrm{N}^{2 s-3}\right)$, and

$$
\int_{\mathrm{SO}(3)}|\mathbf{u}|^{\mathrm{k}} \phi_{\mathrm{N}, \mathrm{s}}(\mathbf{u}) \mathrm{d} \mathbf{u}=\frac{2}{\pi} \int_{0}^{\pi} \phi_{\mathrm{N}, \mathrm{s}}(\omega) \sin ^{2} \frac{\omega}{2} \mathrm{~d} \omega=\mathrm{O}\left(\mathrm{N}^{-\mathrm{k}}\right), \mathrm{k}=0,1, \cdots, 2 \mathrm{~s}-4 .
$$

Following the thought of classical case, we construct the Jackson-type approximate operator as follows, which is different from [1].

$$
J_{N}(f, x)=\int_{G} \phi_{N, s}(\mathbf{u}) \sum_{j=1}^{r}(-1)^{j-1}\left(\begin{array}{l}
r \\
j
\end{array}\right) f\left(\mathbf{u}^{-j} \mathbf{x}\right) d \mathbf{u} .
$$

By the Weyl integral formula (see [10]),

$$
\begin{aligned}
& J_{N}(f, x)=\int_{G} \phi_{N, s}(\mathbf{u}) \sum_{j=1}^{r}(-1)^{j-1}\left(\begin{array}{l}
r \\
j
\end{array}\right) f\left(\mathbf{u}^{-j} \mathbf{x}\right) d \mathbf{u} \\
& =\sum_{j=1}^{r}(-1)^{j-1}\left(\begin{array}{l}
r \\
j
\end{array}\right) \int_{G} \phi_{N, s}(\mathbf{u}) f\left(\mathbf{u}^{-j} \mathbf{x}\right) d \mathbf{u} \\
& =|W(G)|^{-1} \sum_{j=1}^{r}(-1)^{j-1}\left(\begin{array}{l}
r \\
j
\end{array}\right) \int_{T} d(\mathbf{v}) \phi_{N, s}(\mathbf{v}) \int_{G / T} f\left(\mathbf{g v}^{-j} \mathbf{g}^{-1} \mathbf{x}\right) d T d(G / T) \\
& =\left.|W(G)|^{-1}\left|Q^{-1} \sum_{j=1}^{r}(-1)^{j-1}\left(\begin{array}{l}
r \\
j
\end{array}\right) \int_{Q}\right| D(H)\right|^{2} \phi_{N, s}(\mathbf{H}) \int_{G / T} f\left(g \exp (-j \mathbf{H}) \mathbf{g}^{-1} \mathbf{x}\right) d g T d \mathbf{H} \\
& =|W(G)|^{-1}|Q|^{-1} \int_{Q} \int_{G / T} \sum_{j=1}^{r}(-1)^{j-1}\left(\begin{array}{l}
r \\
j
\end{array}\right) \frac{1}{j}|D(H / j)|^{2} \phi_{N, s}(\mathbf{H} / j) f\left(g \exp (-\mathbf{H}) \mathbf{g}^{-1} \mathbf{x}\right) \operatorname{dg} \mathrm{Td} \mathbf{H} \\
& =|W(G)|^{-1}|\mathrm{Q}|^{-1} \int_{Q} \int_{G / T} \sum_{j=1}^{r}(-1)^{j-1}\left(\begin{array}{l}
r \\
j
\end{array}\right) \frac{1}{j}|D(H / j)|^{2} \phi_{N, s}(\mathbf{H} / j) f\left(g \exp (-\mathbf{H}) \mathbf{g}^{-1} \mathbf{x}\right) \mathrm{dgTd} \mathbf{H} \\
& =|\mathrm{W}(\mathrm{G})|^{-1}|\mathrm{Q}|^{-1} \int_{\mathrm{Q}} \int_{\mathrm{G} / \mathrm{T}}|\mathrm{D}(\mathbf{H})|^{2} \mathrm{~K}_{\mathrm{N}}(\exp \mathbf{H}) \mathrm{f}\left(\mathbf{g} \exp (-\mathbf{H}) \mathbf{g}^{-1} \mathbf{x}\right) \mathrm{dg} \mathrm{Td} \mathbf{H} \\
& =|W(G)|^{-1} \int_{T} \int_{G / T} d(\mathbf{v}) K_{N}(\mathbf{v}) f\left(g^{-1} \mathbf{g}^{-1} \mathbf{x}\right) d g T d \mathbf{v} \\
& =\int_{G} K_{N}(\mathbf{u}) f\left(\mathbf{u}^{-1} \chi\right) d u,
\end{aligned}
$$


where

$$
\begin{aligned}
\mathrm{K}_{\mathrm{N}}(\mathbf{u})=\mathrm{K}_{\mathrm{N}}(\mathbf{v})=\mathrm{K}_{\mathrm{N}}(\exp \mathbf{H}) & =\sum_{j=1}^{r}(-1)^{j-1}\left(\begin{array}{l}
\mathrm{r} \\
j
\end{array}\right) \frac{1}{j} \phi_{N, s}(\mathbf{H} / \mathrm{j})\left|\frac{\mathrm{D}(\mathbf{H} / \mathrm{j})}{\mathrm{D}(\mathbf{H})}\right|^{2} \\
& =\sum_{j=1}^{r}(-1)^{j-1}\left(\begin{array}{l}
r \\
j
\end{array}\right) \frac{1}{j} \phi_{N, s}(\omega / j)\left|\frac{\sin (\omega / 2 j)}{\sin \omega / 2}\right|^{2},
\end{aligned}
$$

with $\mathbf{u}=\mathbf{g v g}^{-1}, \mathbf{v} \in \mathrm{T}$, and $\mathbf{v}=\exp \mathbf{H}, \mathbf{H} \in \mathfrak{t}$, and $\mathrm{D}(\mathbf{H})=2 i \sin \frac{\omega}{2}, \mathrm{D}(\mathbf{H} / \mathbf{j})=2 i \sin (\omega / 2 j), d(\mathbf{v})=$ $4 \sin ^{2} \frac{\omega}{2}:=\mathrm{d}(\omega), \exp : \mathfrak{g} \rightarrow \mathrm{G}$ is the exponential map, $\left.\exp \right|_{\mathfrak{t}}: \mathfrak{t} \rightarrow \mathrm{T}$.

Weyl group $W=W(G)$ is defined by $W=N / T$, with $N=\left\{g \in G \mid g T g^{-1}=T\right\}, T$ as above is maximal torus of $\mathrm{G},|\mathrm{W}(\mathrm{G})|=|\mathrm{W}(\mathrm{SO}(3))|=2$ denotes the order of $\mathrm{W}$. $\mathrm{Q}$ is called fundamental domain centered at the origin, which satisfies $\exp (\mathrm{Q})=\mathrm{T}$, here in fact $\mathrm{Q}=\mathbf{t} \cong[-\pi, \pi],|\mathrm{Q}|=2 \pi$ denotes the volume of $\mathrm{Q}$.

Lemma 2.9. If $f \in \mathrm{L}_{p}(\mathrm{SO}(3)), 1 \leqslant p \leqslant+\infty$, then $\mathrm{J}_{\mathrm{N}}(\mathrm{f}) \in \Pi_{\mathrm{N}}$.

Proof. In fact

$$
J_{N}(f, x)=\left(K_{N} * f\right)(\mathbf{x})=\int_{G} K_{N}(\mathbf{u}) f\left(\mathbf{u}^{-1} \mathbf{x}\right) d \mathbf{u}=\int_{G} K_{N}\left(x u^{-1}\right) f(\mathbf{u}) d \mathbf{u} .
$$

Clearly, from (2.4), $\mathrm{K}_{\mathrm{N}}(\mathbf{u})$ is a trigonometric polynomial of degree $\mathrm{N}$, which has not to be an integer.

We write $\mathrm{K}_{\mathrm{N}}(\mathbf{u})=\sum_{l=0}^{N} \sum_{m, n=-l}^{l} c_{m, n}^{l} D_{m, n}^{l}(\mathbf{u})$, and note that (see [4])

$$
\begin{aligned}
D_{m, n}^{l}\left(\mathbf{u}_{1} \mathbf{u}_{2}\right) & =\sum_{k=-l}^{l} D_{m, k}^{l}\left(\mathbf{u}_{1}\right) D_{k, n}^{l}\left(\mathbf{u}_{2}\right), \quad D_{m, n}^{l}\left(\mathbf{u}^{-1}\right)=\left[D_{m, n}^{l}(\mathbf{u})\right]^{-1}=\left[\overline{D_{m, n}^{l}}(\mathbf{u}) \bar{l}=\overline{D_{n, m}^{l}}(\mathbf{u}),\right. \\
J_{N}(f, \mathbf{x}) & =\int_{G} K_{N}\left(\mathbf{x u}^{-1}\right) f(\mathbf{u}) d \mathbf{u}=\sum_{l=0}^{N} \sum_{m, n=-l}^{l} \sum_{k=-l}^{l} c_{m, n}^{l} D_{m, k}^{l}(\mathbf{x}) \int_{G} \overline{D_{n, k}^{l}}(\mathbf{u}) f(\mathbf{u}) d \mathbf{u} .
\end{aligned}
$$

So, $J_{N}(f) \in \Pi_{N}$.

Theorem 2.10. If $\mathrm{f} \in \mathrm{L}_{\mathrm{p}}(\mathrm{SO}(3)), 1 \leqslant p \leqslant+\infty$, then

$$
\left\|f-J_{N}(f)\right\|_{p} \leqslant C \omega_{r}\left(f, \frac{1}{N}\right)_{p},
$$

with $\mathrm{C}>0$ being a constant independent of $\mathrm{f}$ and $\mathrm{N}$.

Proof.

$$
f(\mathbf{x})-J_{N}(f, x)=\int_{G} \phi_{N, s}(\mathbf{u}) \sum_{j=0}^{r}(-1)^{j-1}\left(\begin{array}{l}
r \\
j
\end{array}\right) f\left(\mathbf{u}^{-j} \mathbf{x}\right) d \mathbf{u} .
$$

By Minkowski's inequality and (2.3),

$$
\begin{aligned}
\| f-J_{N}\left(f \|_{p}\right. & \leqslant \int_{G} \phi_{N, s}(\mathbf{u})\left\|\Delta_{h}^{r} f\right\|_{p} d \mathbf{u} \\
& \leqslant \int_{G} \phi_{N, s}() \omega_{r}(f,|\mathbf{u}|)_{p} d \mathbf{u} \\
& \leqslant \omega_{r}(f, 1 / N)_{\mathbf{u p}} \int_{G} \phi_{N, s}(\mathbf{u})(1+N|\mathbf{u}|)^{r} \mathrm{~d} \mathbf{u} \\
& \leqslant C \omega_{r}(f, 1 / N)_{p} .
\end{aligned}
$$

\section{Acknowledgment}

This work was supported by the National Natural Science Foundation of China (No. 11361076). 


\section{References}

[1] D. I. Cartwright, K. Kucharski, Jackson's theorem for compact connected Lie groups, J. Approx. Theory, 55 (1988), 352-359. 1, 2.5, 2

[2] S. Gong, Dianxing qun shangde tiaohe fenxi, (Chinese) [[Harmonic analysis on classical groups]] Chuncui Shuxue yu Yingyong Shuxue Zhuanzhu [Series of Monographs in Pure and Applied Mathematics], Kexue Chubanshe (Science Press), Beijing, (1983). 1

[3] Q.-Z. Han, H.-Z. Sun, Group theory, Peking University Press, Beijing, (1987). 2

[4] R. Hielscher, J. Prestin, A. Vollrath, Fast summation of functions on the rotation group, Math. Geosci., 42 (2010), 773-794. 2.4, 2

[5] D. L. Ragozin, Approximation theory on SU(2), J. Approximation Theory, 1 (1968), 464-475. 2.5

[6] D. Schmid, Marcinkiewicz-Zygmund inequalities and polynomial approximation from scattered data on $\mathrm{SO}(3), \mathrm{Numer}$. Funct. Anal. Optim., 29 (2008), 855-882. 2

[7] M . R. Sepanski, Compact Lie groups, Springer-Verlag, Heidelberg, (2007). 1, 2

[8] J. H. Xie, D. S. Fan, Asymptotic properties of Fourier coefficients on rotation groups, Kexue Tongbao (English Ed.), 32 (1987), 1590-1591. 1

[9] W. T. Xu, X. L. Ka, Group theory and its applications in solid state physics, (Chinese), Higher Education Press, Beijing, (1999). 1

[10] X. A. Zheng, Harmonic analysis on compact homogeneous spaces, (Chinese), Shanghai Scientific and Technical Publishers, (1999). 2.8, 2

[11] X. A. Zheng, Z. F. Xu, H. S. Zhao, Approximation by polynomials on compact Lie groups, I, Best uniform approximation to continuous functions, (Chinese) Adv. in Math. (Beijing), 16 (1987), 61-66. 1

[12] X. A. Zheng, H. S. Zhao, Z. F. Xu, Approximation by polynomials on compact Lie groups, II, Best approximation in the $\mathrm{L}_{p}$ norm, (Chinese) Adv. in Math. (China), 19 (1990), 199-203. 1 